\title{
CÓDIGO ROJO: GUÍA PARA EL MANEJO DE LA HEMORRAGIA OBSTÉTRICA
}

\section{Red code: obstetric haemorrhage management guildeline}

Gladis Adriana Vélez-Álvarez, M.D., * Bernardo Agudelo-Jaramillo, M.D., ** Joaquín

Guillermo Gómez-Dávila, M.D., M.Sc, *** John Jairo Zuleta-Tobón, M.D., M.Sc*****

Recibido: agosto 20/08 - Aceptado: febrero 2/09

\section{RESUMEN}

La hemorragia obstétrica es la primera causa de muerte materna en el mundo. Lo mismo ocurre en el departamento de Antioquia, Colombia. Por ser una situación urgente y que compromete la vida de las mujeres, se presenta la siguiente guía de manejo para el tratamiento del choque hemorrágico de origen obstétrico, basada en los siguientes principios básicos: el manejo óptimo del tiempo, la reposición adecuada del volumen sanguíneo, el trabajo en equipo y la insistencia en el uso de las diferentes maniobras para la disminución de la hemorragia.

Palabras clave: hemorragia, shock hemorrágico, hemorragia posparto, complicaciones del embarazo.

\section{SUMMARY}

Obstetric haemorrhage is the main cause of maternal death around the world. This holds true in the state of Antioquia, Colombia. A management

* Especialista en Obstetricia y Ginecología. Nacer, Salud Sexual y Reproductiva, Facultad de Medicina, Universidad de Antioquia. Medellín, Colombia. Correo electrónico: adriana.velez@medicina.udea. edu.co

** Especialista en Obstetricia y Ginecología. Nacer, Salud Sexual y Reproductiva, Profesor Facultad de Medicina Universidad de Antioquia. Medellín, Colombia.

*** Especialista en Obstetricia y Ginecología, Magíster en epidemiología. Nacer, Salud Sexual y Reproductiva, Profesor Facultad de Medicina Universidad de Antioquia. Medellín, Colombia.

**** Especialista en Obstetricia y Ginecología, Magíster en epidemiología. Nacer, Salud Sexual y Reproductiva, Profesor Facultad de Medicina Universidad de Antioquia. Medellín, Colombia. guide for treating obstetric hemorrhagic shock is thus presented due to the seriousness of this situation compromising women's lives. It was based on the following basic principles: optimum time management, suitable restoration of blood volume, efficient teamwork and insistence on using several techniques for decreasing blood loss.

Key words: haemorrhage, hemorrhagic shock, postpartum haemorrhage, pregnancy complications.

\section{INTRODUCCIÓN}

La hemorragia obstétrica es la primera causa de muerte materna en el mundo. La incidencia varía entre los países, pero globalmente responde al 25\% de las muertes. Aún en los países desarrollados la hemorragia está entre las tres primeras causas de muerte. En Colombia, en el año 2006, la hemorragia posparto fue la segunda causa de muerte después de los fenómenos hipertensivos. ${ }^{1}$ En el departamento de Antioquia, Colombia, la hemorragia obstétrica fue la primera causa de muerte materna durante los años 2004, 2005, 2006 y 2007.

En el año 2006 se publicó un artículo ${ }^{2}$ con los resultados del análisis de las muertes maternas por hemorragia obstétrica, ocurridas en el departamento de Antioquia durante los años 2004 y 2005. En él se resaltaba que la primera causa de hemorragia obstétrica era la hemorragia posparto y que los aspectos más importantes a mejorar tenían que 
ver con la implementación del manejo activo del alumbramiento, el diagnóstico temprano y un tratamiento agresivo del choque y la reanimación.

A partir de estas observaciones el grupo Nacer, Salud Sexual y Reproductiva de la Facultad de Medicina de la Universidad de Antioquia, diseñó un módulo de entrenamiento para la prevención de la morbimortalidad por hemorragia obstétrica, denominado "Código Rojo", en el cual se insiste en la utilización del manejo activo del alumbramiento en todas las pacientes que tengan un parto vaginal institucional y en el manejo secuencial, interdisciplinario, sistemático y agresivo del choque hemorrágico.

Este módulo de entrenamiento se ha aplicado en la ciudad de Medellín, gracias al apoyo de la Secretaría de Salud de Medellín, la Organización Panamericana de la Salud y USAID, y en 7 de las 9 regionales del departamento de Antioquia, financiado por la Dirección Seccional de Salud de Antioquia.

Por ser una situación urgente y que compromete la vida de las mujeres, se presenta la guía "Código Rojo" para el tratamiento del choque hemorrágico de origen obstétrico basada en los siguientes principios básicos: el manejo óptimo del tiempo, la reposición adecuada del volumen sanguíneo, el trabajo en equipo y la insistencia en el uso de las diferentes maniobras para la disminución de hemorragia, como una estrategia para la prevención de la mortalidad materna por hemorragia obstétrica.

\section{OBJETIVO}

Proveer al personal de la salud con una guía para realizar un tratamiento organizado, sistemático, oportuno y pertinente de la hemorragia obstétrica.

\section{Población objeto}

Mujeres gestantes o en el puerperio que presenten choque hipovolémico de origen obstétrico.

\section{Usuarios}

Aplica para médicos generales, enfermeras y especialistas en obstetricia, urgencias y anestesia.

\section{Proceso de elaboración}

Esta guía surgió de la necesidad de contar con un instrumento que sintetizara los aspectos críticos identificados en el análisis de las muertes por hemorragia ocurridas en el departamento de Antioquia. Los puntos que se pretendieron intervenir fueron los siguientes: la falta de un trabajo organizado y sistemático en equipo, el reconocimiento tardío del choque, la falta de un cálculo aproximado de la cantidad de sangre perdida, la reposición inadecuada de cristaloides, la demora en el inicio del suministro de los hemoderivados y un bajo porcentaje de utilización de las maniobras adecuadas para disminuir el sangrado.

\section{Métodos empleados para seleccionar la evidencia}

Se hizo búsqueda libre y controlada en Medline sin restricción de año de publicación, artículos en inglés y en español, búsqueda por tópicos en la biblioteca Cochrane, empleando los términos pertinentes según cada una de las preguntas que se querían responder. Adicionalmente se consultaron las referencias de los artículos encontrados, otras guías del tema y textos de obstetricia y hemorragia obstétrica. El principal criterio de inclusión de los estudios fue el modelo de investigación, para lo cual se estableció un orden jerárquico. Para las preguntas de intervención se partió de metanálisis de ensayos clínicos, o en su defecto, de ensayos clínicos individuales; sin embargo, como se preveía, para varios tópicos no se encontraban ese tipo de estudios, por lo tanto se procedía a realizar búsqueda para estudios de cohorte o de casos y controles y en último lugar estudios descriptivos. Tres de los autores realizaron búsquedas bibliográficas para todas las preguntas planteadas y calificaron la calidad de los artículos. Aquellos que cumplieron los requisitos se discutieron no sólo dentro del grupo de autores sino con otros clínicos, y a partir de estas reuniones se tomaron decisiones por consenso. No se hizo medición objetiva de la concordancia; sin embargo, para la mayoría de preguntas hubo acuerdos de entrada. 


\section{Métodos empleados para calificar la evidencia}

La hemorragia obstétrica es una complicación aguda y urgente que compromete la vida de las mujeres. Esta situación lleva a que muchas de las intervenciones no se puedan someter al rigor metodológico del ensayo clínico controlado y, por lo tanto, muchas de las recomendaciones son opiniones de expertos que se sustentan en los resultados de estudios descriptivos o en extrapolaciones de raciocinios fisiopatológicos. Para la presente guía se emplearon los criterios de los Canadian Task Force on Preventive Health Care $^{3}$ para calificar los niveles de evidencia (tabla 1).

\begin{tabular}{|c|l|}
\hline \multicolumn{2}{|c|}{$\begin{array}{c}\text { Tabla 1. Canadian Task Force } \\
\text { on Preventive Health Care. }\end{array}$} \\
\hline Nivel & $\begin{array}{l}\text { Al menos un ensayo clínico controlado } \\
\text { y aleatorizado. }\end{array}$ \\
\hline I & $\begin{array}{l}\text { Ensayo clínico controlado sin } \\
\text { asignación al azar. }\end{array}$ \\
\hline II-1 & $\begin{array}{l}\text { Estudios de cohortes o casos y } \\
\text { controles. }\end{array}$ \\
\hline II-2 & $\begin{array}{l}\text { Estudios de comparaciones en el } \\
\text { tiempo, con o sin intervención. }\end{array}$ \\
\hline II-3 & Opinión de expertos \\
\hline III &
\end{tabular}

\section{Descripción de los métodos de validación de la guía}

Después de elaborada, especialistas en ginecología y obstetricia integrantes del grupo Nacer y pares internacionales (Canadá y Estados Unidos) revisaron la guía e hicieron sus aportes. Posteriormente se realizó un proceso de validación-capacitación con varios grupos de médicos generales y obstetras, profesionales y auxiliares de enfermería en la ciudad de Medellín, en 7 de las 9 regionales del departamento de Antioquia, y exclusivamente con médicos especialistas y residentes de ginecología y obstetricia en la ciudad de Neiva. La capacitación consistió en la presentación de los aspectos teóricos del "Código Rojo" y luego la realización de ejercicios prácticos en escenarios de simulación, los cuales se adaptaron con ejemplos clínicos acordes al personal que participó en cada taller. En total asistieron 116 médicos generales, 89 enfermeras, 50 auxiliares de enfermería, 35 especialistas en ginecología y obstetricia y 91 estudiantes de medicina. En todos los talleres los asistentes evaluaron por escrito la metodología y los contenidos, hicieron sugerencias de modificación, que se incluyeron según su pertinencia y validez. Posterior a esto se envió la guía a todos los servicios obstétricos del departamento de Antioquia.

\section{DEFINICIONES}

\section{Hemorragia severa}

La definición general es la pérdida de todo el volumen sanguíneo en un período de 24 horas o el sangrado que ocurre a una velocidad superior a $150 \mathrm{~mL} /$ minuto y que, por lo tanto, causa la pérdida del 50\% del volumen en 20 minutos.

Debido a las pérdidas que pueden ocurrir habitualmente en el posparto inmediato, los valores cambian. Se define como hemorragia posparto severa una pérdida estimada de 1000 mL o más o una pérdida menor asociada con signos de choque ${ }^{4}$ (nivel de evidencia III).

\section{Clasificación del choque hipovolémico en la mujer gestante (tabla 2)}

Se utilizó la clasificación clásica de choque hipovolémico de Baskett ${ }^{5}$, pero enfatizando dos aspectos:

- Debido al aumento del volumen plasmático que ocurre en la mujer durante el embarazo, un porcentaje de pérdida dado representa para ella un volumen mayor que en la mujer no embarazada.

- Los parámetros clínicos que se deben evaluar son, en primer lugar, el estado de conciencia y la perfusión, pues las alteraciones en el pulso y la presión arterial son tardíos en la mujer embarazada. 
Tabla 2. Estimación de las pérdidas, de acuerdo a la evaluación del estado de choque.

\begin{tabular}{|c|c|c|c|c|c|c|}
\hline $\begin{array}{c}\text { Pérdida de } \\
\text { volumen (\%) } \\
\text { y mL para } \\
\text { una mujer } \\
\text { embarazada } \\
\text { entre } 50-70 \mathrm{~kg}\end{array}$ & Sensorio & Perfusión & Pulso & $\begin{array}{l}\text { Presión } \\
\text { arterial } \\
\text { sistólica }\end{array}$ & $\begin{array}{c}\text { Grado del } \\
\text { choque }\end{array}$ & $\begin{array}{l}\text { Cantidad de } \\
\text { cristaloides a } \\
\text { reponer en la } \\
\text { primera hora }\end{array}$ \\
\hline $\begin{array}{c}10-15 \% \\
500-1000 \mathrm{~mL}\end{array}$ & Normal & Normal & $60-90$ & Normal & Compensado & Ninguno \\
\hline $\begin{array}{c}16-25 \% \\
1000-1500 \mathrm{~mL}\end{array}$ & $\begin{array}{c}\text { Normal } \\
\text { y/o agitada }\end{array}$ & $\begin{array}{l}\text { Palidez, } \\
\text { frialdad }\end{array}$ & $91-100$ & $80-90$ & Leve & 3000 a $4500 \mathrm{~mL}$ \\
\hline $\begin{array}{c}26-35 \% \\
1500-2000 \mathrm{~mL}\end{array}$ & Agitada & $\begin{array}{l}\text { Palidez, } \\
\text { frialdad más } \\
\text { sudoración }\end{array}$ & $101-120$ & $70-80$ & Moderado & 4500 a $6000 \mathrm{~mL}$ \\
\hline $\begin{array}{c}>35 \% \\
2000-3000 \mathrm{~mL}\end{array}$ & $\begin{array}{l}\text { Letárgica, } \\
\text { inconciente }\end{array}$ & $\begin{array}{l}\text { Palidez, } \\
\text { frialdad más } \\
\text { sudoración } \\
\text { más llenado } \\
\text { capilar >3 } \\
\text { segundos }\end{array}$ & $>120$ & $<70$ & Severo & $>6000 \mathrm{~mL}$ \\
\hline
\end{tabular}

Modificación de Baskett PJF. ABC of major trauma. Management of hypovolemic shock. BMJ 1990;300:1453-7.

\section{Guía para la atención del choque hipovolémico: "Código Rojo"}

\section{Todas las instituciones que atienden pacientes obstétricas deben tener una guía de atención del choque hipovolémico y realizar simulacros periódicamente ${ }^{6}$ (nivel de evidencia III).}

En una situación de emergencia, como la hemorragia obstétrica, es necesario que el equipo asistente esté suficientemente actualizado en las mejores técnicas y estrategias para salvar la vida y que trabaje de manera coordinada y lógica. De manera simultánea debe dirigir los esfuerzos hacia objetivos concretos como salvar a la madre, disminuir la morbilidad que resulta del proceso hemorrágico y de la misma reanimación y en lo posible, salvar al producto de la concepción.

Para lograr implementar el protocolo en una institución se requiere satisfacer los siguientes requisitos:
1. El documento del "Código Rojo" debe estar fácilmente disponible para que sea material de consulta permanente.

2. Se debe garantizar el conocimiento teórico del "Código Rojo" y el entrenamiento adecuado del personal asistencial y de apoyo involucrado. Esta capacitación debe ser permanente y periódicamente se deben realizar simulacros, con el fin de mantener las habilidades y garantizar el entrenamiento del personal nuevo en la institución.

3. Las instituciones deben respaldar el "Código Rojo” con el fin de garantizar los recursos necesarios, adecuar los procesos para evitar bloqueos administrativos y coordinar las actividades con el banco de sangre, el laboratorio y el equipo quirúrgico en las unidades en que estén disponibles.

4. Cada vez que se aplique el "Código Rojo" se debe evaluar su desarrollo y debe surgir información que retroalimente el proceso y contribuya a su mejoramiento y adecuación institucional. 


\section{Principios fundamentales del manejo del choque hipovolémico en la gestante}

Una vez se haga el diagnóstico de choque hipovolémico, el equipo de atención médica debe aplicar los siguientes principios:

1. Priorizar la condición materna sobre la fetal.

2. Siempre trabajar en equipo: es indispensable la comunicación entre el líder y el equipo de trabajo y entre éstos y la familia.

3. Reconocer que el organismo tolera mejor la hipoxia que la hipovolemia; por lo tanto, la estrategia de reanimación del choque hipovolémico en el momento inicial se basa en el reemplazo adecuado del volumen perdido, calculado por los signos y síntomas de choque.

4. Hacer la reposición del volumen con solución de cristaloides, bien sea solución salina 0,9\% o solución de Hartman. Se recomienda el uso de cristaloides porque las soluciones coloidales como almidones, albúmina o celulosa son más costosas y no ofrecen ventajas en cuanto a la supervivencia $^{7,8}$ (nivel de evidencia I).

5. La reposición volumétrica debe ser de $3 \mathrm{~mL}$ de solución de cristaloide por cada $\mathrm{mL}$ de sangre calculado en la pérdida.

6. Las maniobras de monitoreo e investigación de la causa de la hemorragia se deben hacer de manera simultánea con el tratamiento de la misma, en lo posible detener la fuente de sangrado en los primeros 20 minutos.

7. Si al cabo de la primera hora no se ha corregido el estado de choque hipovolémico se debe considerar la posibilidad de que la paciente ya tenga una coagulación intravascular diseminada establecida, porque la disfunción de la cascada de la coagulación comienza con la hemorragia y la terapia de volumen para reemplazo y es agravada por la hipotermia y la acidosis.

8. En caso de que la paciente presente un choque severo la primera unidad de glóbulos rojos se debe iniciar en un lapso de 15 minutos $^{9}$ (nivel de evidencia III).
9. Se puede iniciar con glóbulos rojos "O negativo" y/o sangre tipo específica sin pruebas cruzadas hasta que la sangre tipo específica con pruebas cruzadas esté disponible. Si no hay glóbulos rojos "O negativo" disponibles se pueden utilizar glóbulos rojos "O positivo"10,11 (nivel de evidencia II-3).

\section{Secuencia temporal del "Código Rojo" (ver anexo 1):}

Todo el equipo de salud que atiende pacientes obstétricas debe estar familiarizado con los signos clínicos del choque.

\section{Minuto Cero: activación del "Código Rojo"}

En general, la pérdida de $500 \mathrm{~mL}$ rara vez compromete la salud materna. Parece que la percepción de la pérdida de más de 1000 mL es un buen punto de corte para iniciar las maniobras de reanimación.

La activación del "Código Rojo" la realiza la primera persona del equipo asistencial que establece contacto con la paciente que sangra y evalúa los parámetros descritos en la tabla 2. Determina en su orden: estado del sensorio, perfusión, pulso y presión arterial.

El grado de choque lo establece el peor parámetro encontrado. Ante signos de choque y/o un cálculo de sangrado superior a 1000 mL se debe activar el "Código Rojo"” (nivel de evidencia III).

La activación del "Código Rojo" puede ocurrir en el servicio de urgencias, en hospitalización o en las salas de cirugía, partos o recuperación, y por lo tanto, se debe definir un mecanismo rápido y eficiente para la activación del mismo, garantizando que alerte al personal involucrado sin necesidad de realizar múltiples llamadas; se sugiere que sea por altavoz. 
En el minuto cero la activación del "Código Rojo" debe desencadenar varias acciones en forma automática:

- Alertar al servicio de laboratorio y/o banco de sangre, si éste se encuentra disponible en la institución.

- Alertar al servicio de transporte. Si la ambulancia está en la institución debe permanecer allí mientras se resuelve el "Código Rojo", y si no está, se debe contactar al centro regulador de remisiones para avisar que se tiene un "Código Rojo" en proceso.

- Iniciar el calentamiento de los líquidos para uso venoso a $39^{\circ} \mathrm{C}(1000 \mathrm{~mL}$ en microondas o con electrodo en agua, se logra en 2 minutos). Debe estar claramente definido el funcionario que empezará a calentarlos (la hipotermia aumenta el riesgo de falla orgánica y coagulopatía).

- Desplazamiento del mensajero u otra persona definida previamente que asuma tal función al servicio donde se activó el "Código Rojo”.

\section{Minutos 1 a 20: reanimación y diagnóstico}

La resucitación inicial en el choque hemo-
rrágico incluye la restauración del volumen
circulante, mediante la aplicación de dos
catéteres gruesos y la infusión rápida de
soluciones cristaloides
cia I).

Después de activado el "Código Rojo" el equipo debe realizar las siguientes acciones:

- Posicionarse en el sitio asignado para el cumplimiento de sus funciones.

- Iniciar el suministro de oxígeno a la paciente, garantizando la máxima FIO2 con máscara reservorio, Ventury al 35-50\% o cánula nasal a 4 litros por minuto.

- Canalizar dos venas con catéteres \#14 (café marrón garantiza un flujo de $330 \mathrm{~mL} / \mathrm{min}$ ) o \#16 (gris: $225 \mathrm{~mL} / \mathrm{min}$ ). Tomar muestras en tres tubos de tapa roja, morada y gris, para hemoglobina, (Hb), hematocrito (Hcto), plaquetas, hemoclasificación, pruebas cruzadas, tiempo de protrombina (TP), tiempo parcial de tromboplastina (TPT) y fibrinógeno (según el nivel de complejidad). Si el sitio inicial de atención no cuenta con laboratorio, estas muestras se deben conservar y enviar debidamente identificadas cuando se transfiera la paciente a otra institución. Iniciar la administración de 2000 mL de solución salina normal o Hartman ${ }^{12}$ (nivel de evidencia III). Clasificar el grado de choque y complementar el volumen de líquidos requerido de acuerdo al estado de choque (se debe tener precaución en las pacientes con preeclampsia y con cardiopatías).

- Identificar la causa de sangrado e iniciar su manejo. En el primer trimestre, descartar el aborto y sus complicaciones, la gestación ectópica y la mola hidatidiforme; en el segundo y tercer trimestre, identificar las causas placentarias como placenta previa, abrupcio de placenta y la posibilidad de ruptura uterina; $y$ en el posparto, utilizar la nemotecnia de las 4 "T": tono (atonía, $70 \%$ de los casos), trauma (desgarros del canal vaginal 20\%), tejido (retención de restos placentarios 10\%) y trombina (coagulopatía 1\%). Igualmente se debe descartar la ruptura uterina. En los sitios donde no se dispone del recurso de anestesiólogo se recomienda el uso de meperidina y diazepam para realizar las maniobras de revisión del canal (tabla 3). El medicamento de elección para el manejo de la atonía es la oxitocina administrada en infusión. Si no hay contraindicaciones se debe administrar metergina simultáneamente. Si con estos medicamentos no se logra el control se debe adicionar misoprostol (tabla 3).

- Evacuar la vejiga y medir la eliminación urinaria permanentemente. Mantener la temperatura corporal estable cubriendo a la paciente con frazadas. En choque severo, iniciar la transfusión de 2 unidades de glóbulos rojos (GR) "O negativo". 


\begin{tabular}{|c|c|c|}
\hline Medicamento & Dosis & Comentario \\
\hline Líquidos de sostenimiento & $150-300 \mathrm{cc} /$ hora & $\begin{array}{l}\text { Solamente cuando se controle la } \\
\text { hemorragia }\end{array}$ \\
\hline Meperidina & $0,5-1 \mathrm{mg} / \mathrm{kg}$ & \multirow{2}{*}{$\begin{array}{c}\text { Si se requiere sedación para realizar los } \\
\text { procedimientos }\end{array}$} \\
\hline Diazepam & $0,05-0,1 \mathrm{mg} / \mathrm{kg}$ & \\
\hline Oxitocina & $\begin{array}{c}\text { 40-120 mU/min, se puede aumentar hasta } \\
200 \mathrm{mU} / \mathrm{min}: 20 \mathrm{U} / 500 \mathrm{cc} \text { SSN pasar } \\
\text { desde } 60 \text { a } 300 \mathrm{~mL} / \text { hora }\end{array}$ & $\begin{array}{c}\text { Dosis máxima: } 6 \text { ampollas en } 24 \mathrm{~h} \\
\text { (60 U) }\end{array}$ \\
\hline $\begin{array}{l}\text { Misoprostol } \\
\text { (prostaglandina sintética) }\end{array}$ & 800-1000 $\mu \mathrm{g}$ /vía rectal & $\begin{array}{l}\text { Utilizarlo como medicamento de segunda } \\
\text { línea luego de la oxitocina }\end{array}$ \\
\hline $\begin{array}{l}\text { Metilergonovina } \\
\text { Methergina }\end{array}$ & $\begin{array}{l}0,2 \text { mg IM, repetir en } 20 \text { minutos. Se } \\
\text { puede continuar cada } 4-6 \text { horas }\end{array}$ & $\begin{array}{l}\text { Máximo } 5 \text { ampollas (1 mg) } \\
\text { en } 24 \text { horas. } \\
\text { Contraindicado en hipertensión, migraña, } \\
\text { coronariopatía }\end{array}$ \\
\hline Dopamina & $\begin{array}{l}200 \text { mg en } 500 \text { cc de SS 0,9\%. } \\
\text { Iniciar mínimo } 6 \text { mg/kg/min }\end{array}$ & No iniciar con dosis menores \\
\hline $\begin{array}{l}\text { Plasma fresco } \\
(1 \mathrm{U}: 250 \mathrm{cc})\end{array}$ & $12-15 \mathrm{cc} / \mathrm{kg}$ & $\begin{array}{l}\text { Utilizar si PT o TPT } \\
\text { son superiores a } 1,5\end{array}$ \\
\hline Plaquetas & $1 \mathrm{U}$ : aumenta 8000 a 10000 plaquetas & $\begin{array}{l}\text { Mantener por encima } \\
\text { de } 50.000 \text { plaquetas /cc }\end{array}$ \\
\hline $\begin{array}{l}\text { Crioprecipitado } \\
(1 \mathrm{U}: 50-60 \mathrm{cc})\end{array}$ & $1-2 \mathrm{~mL} / \mathrm{kg}$ & $\begin{array}{l}\text { Utilizar si el fibrinógeno es menor } \\
\text { de } 100 \mathrm{mg} / \mathrm{dL}\end{array}$ \\
\hline
\end{tabular}

Si no hay disponible, iniciar "O Rh positivo". Definir si se puede continuar con el manejo del caso en la institución o si requiere remisión, según la causa sospechada o definida y el nivel de atención en el que se encuentre. Mantener informada a la familia y, de acuerdo a las condiciones, contar con su eventual colaboración en algunos aspectos de la atención o de la remisión.

\section{En el manejo del choque hemorrágico es necesaria la rápida identificación de la causa y su control, mientras se realizan las medidas iniciales de reanimación.}

\section{Tiempo 20 a 60 minutos: estabilización}

- En choque grave, iniciar la transfusión de sangre tipo específica sin pruebas cruzadas.

- Conservar el volumen útil circulante: si el estado de choque persiste, se debe mantener el reemplazo de líquidos necesarios para mejorar la perfusión y la recuperación hemodinámica. Una vez cese la hemorragia y se controle el choque se debe mantener la infusión de cristaloides de 150 a $300 \mathrm{~mL} /$ hora. Es prudente vigilar los pulmones mediante la auscultación frecuente en busca de signos de edema agudo por sobrecarga de volumen, que en caso de presentarse, se debe tratar con las medidas clásicas para el mismo.

- Si el diagnóstico es una atonía se deben mantener las maniobras compresivas, como el masaje uterino bimanual o la compresión externa de la aorta y el suministro de uterotónicos (tabla 3).

- Garantizar la vigilancia de los signos de perfusión como el estado de conciencia, el llenado capilar, el pulso, la presión arterial y la eliminación urinaria. Además se debe vigilar la frecuencia respiratoria.

- Si después de la reposición adecuada de volumen la paciente continúa hipotensa, hay que considerar 
la utilización de medicamentos inotrópicos y vasoactivos (tabla 3).

- Evaluar, de acuerdo a la situación clínica y al nivel de atención en el que se encuentre la paciente, si puede continuar el tratamiento o es mejor trasladarla a otra institución.

- Si el sangrado es por atonía y su control es difícil, y se cuenta con los recursos suficientes para realizar un procedimiento quirúrgico, se debe alcanzar un estado óptimo de perfusión antes de realizarlo. Es necesario definir la necesidad de realizar procedimientos, desde el menos hasta el más agresivo: la prueba de taponamiento uterino, las suturas de B-Lynch, la ligadura selectiva de las arterias uterinas y, por último, la histerectomía.

- Mantener adecuadamente informada a la familia.

\section{Una resucitación adecuada requiere la eva- luación continua de la respuesta, mediante la vigilancia de los signos clínicos y los con- troles seriados hematológicos, bioquímicos y metabólicos.}

\section{Tiempo 60 minutos: manejo avanzado}

Después de una hora de hemorragia e hipoperfusión, con o sin tratamiento activo, existe una alta probabilidad de una coagulación intravascular diseminada (CID). Antes de realizar cualquier procedimiento quirúrgico es necesario garantizar la recuperación de la coagulación. Se debe proceder así:

- Establecer la vigilancia avanzada para controlar la CID con la reevaluación de las pruebas de coagulación como el TPT, TP, el fibrinógeno y el dímero D.

- Considerar siempre la decisión crítica: si se asume el manejo de la paciente se debe disponer de los recursos quirúrgicos, los hemoderivados y la vigilancia en una unidad de cuidado intensivo (UCI). Si no se tienen estas condiciones se debe trasladar a la paciente en óptimas condiciones a un nivel superior (nivel III o IV).
- Si el sangrado continúa en instituciones con el recurso del especialista y la tecnología adecuada, se debe definir la posibilidad de intervenciones avanzadas como la embolización selectiva o la realización de los procedimientos quirúrgicos, si éstos no se han realizado aún (ligadura de las arterias uterinas, sutura de B-Lynch, histerectomía).

- En lo posible, solicitar asesoría del hematólogo para el manejo de la CID.

- Siempre se debe corregir la CID antes de realizar procedimientos quirúrgicos:

- Garantizar que el recuento de plaquetas sea superior a 50.000/mL. Cada unidad de plaquetas de $50 \mathrm{~mL}$ aporta 5000 - 8000 plaquetas por mL.

- Utilizar el plasma fresco congelado si los valores de TP y/o del TPT son 1,5 veces mayores al del control. Dosis: 12-15 mL/kg. Cada unidad de $250 \mathrm{~mL}$ aporta $150 \mathrm{mg}$ de fibrinógeno y otros factores de la coagulación. La decisión de aplicar el plasma fresco no depende directa y exclusivamente de la cantidad de glóbulos suministrados, como se recomendaba anteriormente. ${ }^{13}$

- El crioprecipitado se debe utilizar si el fibrinógeno es inferior a $100 \mathrm{mg} / \mathrm{dL}$ y/o el TPT y el TP no se corrigen con la administración del plasma fresco. Una unidad de $10 \mathrm{a} 15 \mathrm{~mL}$ aporta $200 \mathrm{mg}$ de fibrinógeno, 100 unidades de factor VIII, 80-100 U de factor de von Willebrand, 50-100 U de factor XIII y fibronectina. Dosis: $2 \mathrm{~mL} / \mathrm{kg}$ de peso.

- Evaluar el estado ácido-básico, los gases arteriales, el ionograma y la oxigenación. Recordar que la coagulación es dependiente del estado ácido-básico, de la oxigenación y de la temperatura.

- Conservar el volumen útil circulatorio apoyándose siempre en los criterios clínicos de choque.

- Mantener las actividades de hemostasia y la vigilancia estricta del sangrado mientras se traslada la paciente a otra institución o se lleva a cirugía.

- Mantener adecuadamente informada a la familia. 


\section{Siempre que se defina el traslado:}

Garantizar el transporte adecuado, de preferencia en ambulancia medicalizada, con el equipo humano acompañante entrenado en "Código Rojo", que continúe durante el desplazamiento con la aplicación del mismo, con énfasis en el masaje activo, las maniobras de reanimación, reemplazo de volumen y administración de medicamentos (goteo de oxitocina en caso de atonía). En la ambulancia se debe disponer del equipo de reanimación cardiopulmonar, medicamentos, infusores de líquidos y oxígeno.

Acompañar a la familia con una explicación clara y objetiva, que proceda de quien asume el proceso de coordinación del "Código Rojo".

\section{Organización del equipo de trabajo}

Con el fin de evitar el caos durante la asistencia de una situación crítica, como la hemorragia obstétrica con choque hipovolémico, es necesario organizar al equipo humano disponible asignando funciones específicas, las cuales se deben conocer y estudiar previamente en los entrenamientos institucionales. Esta organización del equipo mejora las condiciones de trabajo y facilita la realización de las funciones para la recuperación y mantenimiento de la vida de la mujer en choque. Cada miembro participante en el "Código Rojo" debe cumplir sus funciones con el fin de evitar la duplicación o la falta de realización de alguna de ellas. La distribución que se propone considera como ideal que se disponga de cuatro personas. Será necesario adaptarla de acuerdo a la disponibilidad del equipo humano de cada institución. El personal debe estar claramente asignado para que en cada turno cada persona conozca su responsabilidad. Tener disponible un sistema de tarjetas del "Código Rojo" ayuda al cumplimiento de los objetivos.

- Responsables del "Código Rojo": ver tarjetas con descripción de funciones en el anexo 2.

- Coordinador del "Código Rojo": debe ser médico general de la institución o especialista en ginecología y obstetricia, siempre y cuando esté disponible. En caso de no estar presente el médico, debe ser la persona con mayor experiencia en el manejo de esta situación. Esta persona se debe ubicar hacia la parte inferior del cuerpo de la paciente, al lado de la pelvis o de frente a la región perineal, según sea el caso, para realizar las maniobras necesarias de control del sangrado. Además debe ocuparse de vigilar el cumplimiento del "Código Rojo”, realizar los diagnósticos y procedimientos, así como ordenar los medicamentos.

- Asistente 1: puede ser un médico, enfermera profesional o una auxiliar de enfermería. Se encarga de informar a la paciente acerca de la condición y el procedimiento a realizar, así como de garantizar la vía aérea permeable, monitorizar los signos vitales, cubrir a la paciente con frazadas y llevar el registro clínico del "Código Rojo" (ver anexo 3).

- Asistente 2: puede ser un médico, enfermera profesional o una auxiliar de enfermería. Se encarga de garantizar permanentemente el acceso venoso.

- Circulante(s): puede ser una auxiliar de enfermería u otra persona de la institución que esté capacitada para su función.

\section{Escenarios específicos del choque hipovolémico}

1. Paciente que mejora en los primeros $20 \mathrm{mi}-$ nutos con las maniobras iniciales

- Continuar la vigilancia estricta:

- Evaluar signos de perfusión (sensorio, llenado, pulso y PA) mínimo cada 15 minutos durante las siguientes dos horas.

- Vigilar el sangrado vaginal mínimo cada 15 minutos durante las siguientes 4 horas.

- Si fue una hemorragia por atonía uterina, vigilar la contracción uterina y el sangrado vaginal mínimo cada 15 minutos por las siguientes 2 horas.

- Soporte estricto:

- Continuar los líquidos IV a razón de 150 $300 \mathrm{~mL} /$ hora.

- Si fue una atonía, continuar los uterotónicos por 12 a 24 horas.

- Mantener la oxigenación. 
- Definir la necesidad de transfusión (hemoglobina $<7 \mathrm{~g} / \mathrm{dL}$ ).

2. Paciente que no mejora con las maniobras iniciales

- Continuar la vigilancia estricta de signos vitales y signos de choque mientras se define el lugar de la atención.

- Continuar con el masaje uterino y los uterotónicos, en los casos pertinentes, durante el período previo a la cirugía o durante la remisión.

- Definir el nivel de atención.

3. Paciente que mejora en la reanimación inicial, pero recae en choque posteriormente

- Repasar todos los procesos de los tiempos 1 a 20, 20 a 60 y ubicarse en el tiempo que calcula para la paciente (pensar siempre en la posibilidad de CID).

- Definir el nivel de atención: si no la remite, debe aplicar vigilancia avanzada: coagulación, estado ácido-básico, ionograma.

- Definir conducta quirúrgica, previa estabilización, intentando alcanzar la máxima estabilidad posible.

- Garantizar el soporte posquirúrgico avanzado: unidad de cuidados intensivos y trombo profilaxis, una vez resuelta la hemorragia y la CID.

4. Paciente menor de edad, testigo de Jehová o hija de padres seguidores de este credo religioso

- Realizar el manejo integral del "Código Rojo" y utilizar los hemoderivados cuando estén indicados sin considerar la determinación religiosa de la paciente o de los padres, ya que predomina el cuidado de la salud sobre los condicionantes religiosos. ${ }^{14,15,16}$

- Existe confusión en el medio con respecto a las implicaciones legales de tomar esta decisión. Las sentencias de la corte han sido claras al respecto: "El primero de los derechos fundamentales es el derecho a la vida. Es un derecho inherente al individuo, lo que se pone de presente en el hecho de que sólo hay que existir para ser titular del mismo. De otra parte, no se puede ser titular de derechos sin la vida presente, pasada o fu- tura. Así, resulta la vida un presupuesto para el ejercicio de los demás derechos". ${ }^{14}$ "No se trata de desconocer la condición de autonomía relativa del menor adulto, se trata de evitar que sin pretexto de la misma, el menor, en condiciones extremas de vulnerabilidad (peligro inminente de muerte), adopte decisiones que atenten contra él mismo y contra su integridad". "Las creencias religiosas de la persona no pueden conducir al absurdo de pensar que, con fundamento en ellas, se pueda disponer de la vida de otra persona, o de someter a grave riesgo su salud y su integridad física, máxime, como ya se dijo, cuando se trata de un menor de edad, cuya indefensión hace que el estado le otorgue una especial protección, de conformidad con el artículo 13 superior. Por lo demás, es de la esencia de las religiones en general, y en particular de la cristiana, el propender por la vida, la salud corporal y la integridad física del ser humano; por ello, no deja de resultar paradójico que sus fieles invoquen sus creencias espirituales, como ocurre en este caso, para impedir la oportuna intervención de la ciencia en procura de la salud de una hija menor. No existe pues, principio de razón suficiente que pueda colocar a un determinado credo religioso en oposición a derechos tan fundamentales para un individuo como son la vida y la salud". ${ }^{16}$

- No se recomienda la utilización rutinaria e inicial de sustitutos sanguíneos, ni en éstas ni en otras pacientes, ya que no se ha encontrado suficiente información en el manejo obstétrico urgente, se sustentan en reporte de casos, no están disponibles en la mayoría de las instituciones donde se aplica el "Código Rojo" y, en otros contextos, se ha encontrado que algunos de ellos pueden aumentar los riesgos para las pacientes. ${ }^{17}$

\section{REFERENCIAS}

1. Departamento Administrativo Nacional de Estadística, DANE. Estadísticas vitales. Bogotá D.C.: DANE. Visitado en 2008 Jun 20. Disponible en:http:/www. dane.gov.co/index.php?option $=$ com_content\&task $=$ category\&sectionid $=16 \& \mathrm{id}=36 \&$ Itemid $=148$ 
2. Vélez AG, Gómez DJ, Zuleta TJ. Análisis de las muertes maternas por hemorragia en el departamento de Antioquia, Colombia: años 2004 y 2005. Rev Colomb Obstet Ginecol 2006; 57:147-55.

3. Canadian Task Force on Preventive Health Care. Levels of Evidence - Research Design Rating. Toronto: CTFPHC; c1997. Visitado en 2008 Jun 20. Disponible en: http://ctfphc.org/ctfphc\&methods.htm\#Table_2

4. Ramanathan G, Arulkumaran S. Postpartum haemorrhage. Current Obstetrics Gynaecology 2006;16:6-13.

5. Baskett PJ. ABC of major trauma. Management of hypovolaemic shock. BMJ 1990;300:1453-7.

6. Tipples M, Paterson BS. Labor ward drills. En: B-Lynch C, Keith Lg, Lalonde AB, Karoshi M, editors. A textbook of postpartum hemorrhage: a comprehensive guide to evaluation, management and surgical intervention. Londres: Sapiens Publishing; 2006. p. 127-35.

7. Perel P, Roberts I. Colloids versus crystalloids for fluid resuscitation in critically ill patients. Cochrane Database of Systematic Reviews 2007;(4):CD000567. DOI: 10.1002/14651858.CD000567.pub3.

8. Choi PT, Yip G, Quinonez LG, Cook DJ. Crystalloids vs. colloids in fluid resuscitation: a systematic review. Crit Care Med 1990;27:200-10.

9. Macphail S, Kate T. Massive postpartum haemorrhage and management of disseminated intravascular coagulation. Curr Obstet Gynaecol 2004;14:123-31.

10. Cortés A, Wedekin W, Bolaños F. Reanimación con glóbulos rojos $\mathrm{Rh}$ positivos y sin prueba cruzada en emergencias médicas. Colombia Médica 2004;35:185-90.

11. Keith L, Berger G, Pollack W. The transfusion of Rhpositive blood into negative women. Am J Obstet Gynecol 1976;125:502-6.

12. Santoso JT, Saunders BA, Grosshart K. Massive blood loss and transfusion in obstetrics and gynecology. Obstet Gynecol Surv 2005; 60:827-37.

13. Erber AN, Perry DJ. Plasma and plasma products in the treatment of massive haemorrhage. Best Pract Res Clin Haematol 2006;19:97-112.
14. Colombia, Corte Constitucional, Sala Quinta de revisión, T-452/92, 10 de julio de 1992, M. P. Fabio Morón Díaz, expediente T 1429.

15. Colombia, Corte Constitucional, Sala Octava de revisión, revisión de tutelas T-474/96, 25 de septiembre de 1996. M. P. Fabio Morón Díaz, expediente T 100472.

16. Colombia, Corte Constitucional, Sala Novena de revisión, T-411/94, 19 de septiembre de 1994. M. P. Vladimiro Naranjo Mesa, expediente T 38362.

17. Natanson C, Kern SJ, Lurie P, Banks SM, Wolfe SM. Cell-free hemoglobin-based blood substitutes and risk of myocardial infarction and death: a meta-analysis. JAMA 2008;299:2304-12.

\section{BIBLIOGRAFÍA RECOMENDADA}

- Bonnar J, Massive obstetric haemorrhage. Bailliere's Best Res Clin Obstet Gynaecol 2000;14:1-18.

- Hofmeyr GJ, Mohlala BK. Hypovolaemic shock. Best Pract Res Clin Obstet Gynaecol 2001;15:645-62.

- Jansen AJ, van Rhenen DJ, Steegers EA, Duvekot JJ. Postpartum hemorrhage and transfusion of blood and blood components. Obstet Gynecol Surv 2005;60:663-71.

- Mousa HA, Alfirevic Z. Treatment for primary postpartum haemorrhage. Cochrane Database Syst Rev 2007;(1):CD003249.

- Tsu VD, Langer A. Aldrich T. Postpartum hemorrhage in developing countries: is the public health community using the right tools? Int J Gynaecol Obstet 2004;85: S42-51.

- World Health Organization. Guidelines for emergency management of major obstetric haemorrhage. En: World Health Organization. The clinical use of blood. Geneva: WHO; 2002. p. 222.

- World Health Organization. Manejo de las complicaciones del embarazo y del parto: guía para obstetrices y médicos. WHO/RHR/00.7. Traducción al español por la Organización Panamericana de la Salud. 2002. 
Anexo 1. Afiche con el flujograma del "Código Rojo"

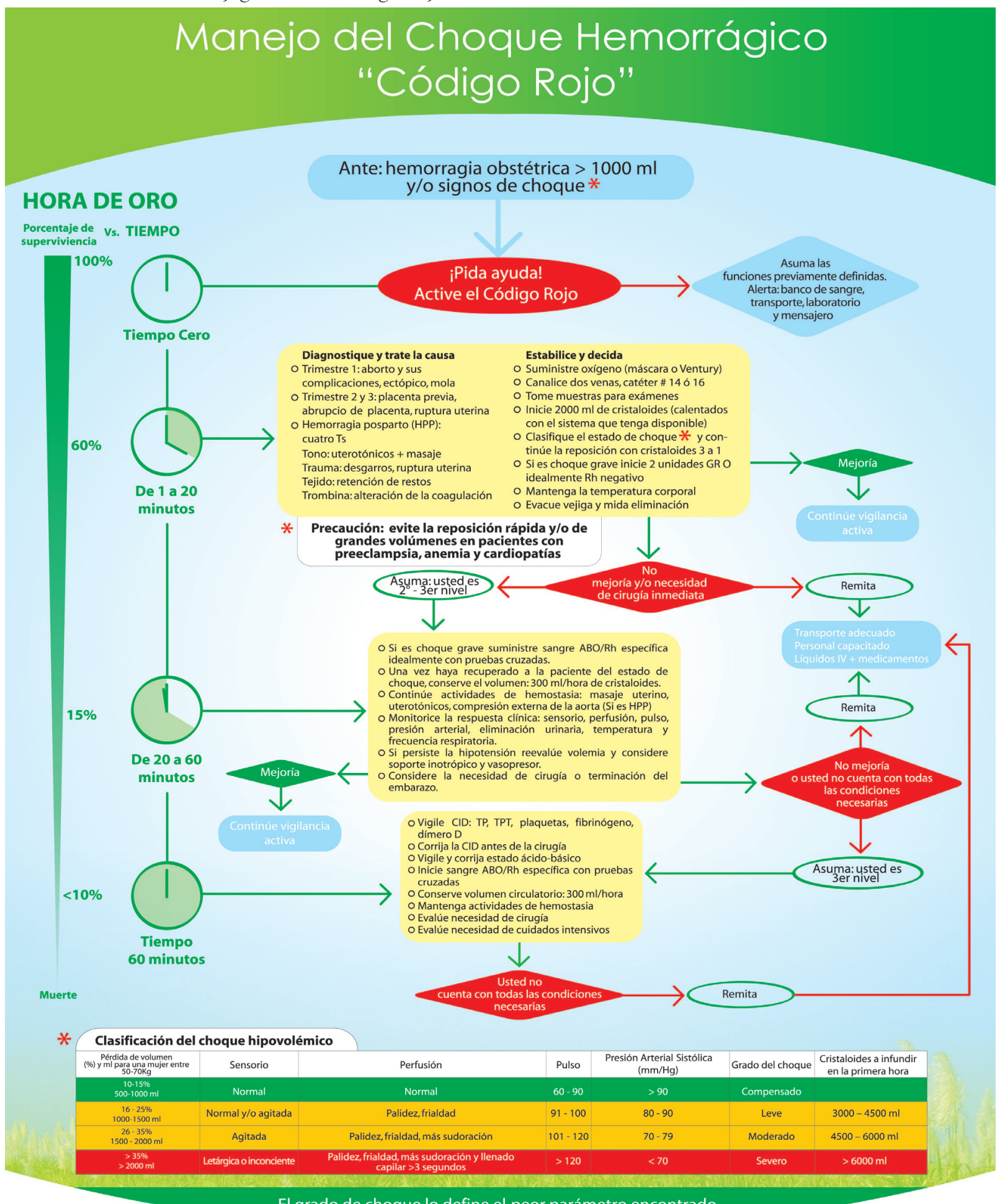

El grado de choque lo define el peor parámetro encontrado

$\underset{\text { Ministerio de la Protección Social }}{\text { Mepublica de Colombia }}$
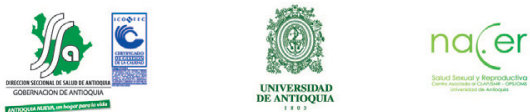
Anexo 2. Tarjetas para la asignación de funciones.

\section{Coordinador (médico)}

Posicionado hacia la parte inferior del cuerpo de la paciente, al lado de la pelvis o de frente a la región perineal

- Busque la causa de choque hemorrágico e inicie el tratamiento de la misma.

- Trimestre 1: aborto y sus complicaciones, ectópico, mola.

- Trimestre 2 y 3: placenta previa, abrupcio de placenta, ruptura uterina.

- En hemorragia posparto: causas basadas en el protocolo de la "cuatro T": tono, trauma, tejido y trombina.

- Evacue la vejiga y deje sonda Foley.

- Tome la decisión temprana de remisión o de asumir el caso, de acuerdo a la causa y al nivel de atención en el que se encuentre.

- Verifique continuamente que los asistentes cumplan sus funciones y defina los cambios a que haya lugar.

- Ordene la aplicación de los medicamentos necesarios.

- Clasifique el estado de choque y su evolución en el tiempo de reanimación y el reemplazo de volumen.

- Envíe la información requerida para los familiares o acompañantes por medio de la persona asignada a esta función.

\section{Asistente 1}

Posicionarse a la cabecera de la paciente, explicarle los procedimientos a seguir y brindarle confianza

- Suministre oxígeno suplementario: máscara o Ventury a 35 - 50\% o cánula nasal a 4 litros/minuto.

- Si la paciente está embarazada con más de 20 semanas, verifique que esté en posición con desviación uterina a la izquierda. No aplica posparto.

- Anote los eventos en la hoja de registro del "Código Rojo".

- Tome la presión arterial y el pulso.

- Monitorice con oximetría de pulso si está disponible.

- Registre la temperatura y cubra a la paciente con cobijas para evitar la hipotermia.

- Reevalué el estado de choque luego de la infusión de los líquidos e informe al coordinador.

- Colabore con el coordinador en la realización de procedimientos si así se requiere.

\section{Asistente 2}

Posicionarse al lado izquierdo de la paciente

- Garantice acceso y funcionamiento de 2 vías venosas con catéter \# 14 ó 16: tome muestras sanguíneas (tres tubos) y coloque 2 litros de cristaloides calientes.

- Realice las órdenes de laboratorio necesarias para hemoglobina, hematocrito, plaquetas, TP, TPT, fibrinógeno, pruebas cruzadas y clasificación sanguínea. En instituciones de alta complejidad, también para dímero $\mathrm{D}$, ionograma y ph y gases arteriales, cuando se requieran.

- En choque severo: solicite 2 U de glóbulos rojos "O” idealmente Rh negativo.

- Aplique los líquidos y medicamentos ordenados por el coordinador.

\section{Circulante}

- Inicie el calentamiento de los líquidos, inicialmente $2000 \mathrm{~mL}$ a $39^{\circ} \mathrm{C}$.

- Marque los tubos de muestras sanguíneas, garantice que las muestras sean recogidas por el patinador, lleguen al laboratorio y se inicie su procesamiento.

- Llame a más personas, de acuerdo al requerimiento del coordinador.

- Puede ser requerido por el coordinador para asistir en algún procedimiento (revisión del canal).

- Establezca contacto con la familia y manténgala informada: la información la define el coordinador. 
Anexo 3. Formato de seguimiento del manejo del choque hipovolémico - "Código Rojo"

\begin{tabular}{|c|c|c|c|c|c|c|c|c|c|}
\hline $\begin{array}{l}\text { Nombre de la } \\
\text { paciente }\end{array}$ & & & & & \multicolumn{2}{|c|}{$\begin{array}{l}\text { Número de } \\
\text { historia }\end{array}$} & & $\begin{array}{l}\text { Fecha de la } \\
\text { atención }\end{array}$ & $\begin{array}{l}\square \square \text { - } \square \square \text { - } \square \square \\
\text { Día - mes - año }\end{array}$ \\
\hline $\begin{array}{l}\text { Hora de } \\
\text { ingreso }\end{array}$ & & \multicolumn{3}{|c|}{$\begin{array}{c}\text { Hora de } \\
\text { activación }\end{array}$} & \multirow{3}{*}{\multicolumn{2}{|c|}{ Procedencia }} & Hospitalizada $\quad \square$ & \multicolumn{2}{|l|}{ Sala: } \\
\hline Administrador & & & & & & & Remitida $\quad \square$ & \multicolumn{2}{|l|}{ Lugar: } \\
\hline Asistente 1 & & & & & & & Propios medios $\square$ & \multicolumn{2}{|l|}{ EPS/ARS: } \\
\hline Asistente 2 & & & & & \multirow{2}{*}{\multicolumn{2}{|c|}{$\begin{array}{l}\text { Contacto } \\
\text { al grupo de } \\
\text { apoyo }\end{array}$}} & \multirow{2}{*}{\multicolumn{3}{|c|}{$\begin{array}{l}\text { Banco de sangre } \square \text { Hematólogo } \square \text { Neonatólogo } \square \text { UCE/UCI } \square \\
\text { Cirujano } \square \text { Anestesiólogo } \square \text { Nivel } 2 \square \text { Nivel } 3 \square\end{array}$}} \\
\hline Acompañante & & & & & & & & & \\
\hline \multirow{2}{*}{$\begin{array}{l}\text { Información } \\
\text { general de la } \\
\text { paciente }\end{array}$} & \multirow[b]{2}{*}{ Edad } & \multirow{2}{*}{ ०० } & \multirow{2}{*}{$\begin{array}{c}\text { Edad } \\
\text { gestacional }\end{array}$} & \multirow{2}{*}{ ๑० } & \multirow{2}{*}{$\begin{array}{c}\text { Peso } \\
(\mathrm{P})\end{array}$} & \multirow[b]{2}{*}{$\square \square$} & \multirow{2}{*}{ Código activado por } & \multicolumn{2}{|l|}{ Nombre } \\
\hline & & & & & & & & Cargo & \\
\hline
\end{tabular}

\begin{tabular}{|c|c|c|c|c|c|}
\hline $\begin{array}{l}\text { Pérdida de volumen } \\
\text { (\%) y mL para una } \\
\text { mujer de } 50-70 \mathrm{~kg}\end{array}$ & Sensorio & Perfusión & Pulso & $\begin{array}{l}\text { Presión arterial } \\
\text { sistólica }\end{array}$ & Grado del choque \\
\hline $\begin{array}{c}10-15 \% \\
500-1000 \mathrm{~mL}\end{array}$ & Normal & Normal & $60-90$ & Normal & Compensado \\
\hline $\begin{array}{c}16-25 \% \\
1000-1500 \mathrm{~mL}\end{array}$ & $\begin{array}{c}\text { Normal } \\
\text { y/o agitada }\end{array}$ & Palidez, frialdad & $91-100$ & $80-90$ & Leve \\
\hline $\begin{array}{c}26-35 \% \\
1500-2000 \mathrm{~mL}\end{array}$ & Agitada & $\begin{array}{l}\text { Palidez, frialdad más } \\
\text { sudoración }\end{array}$ & $101-120$ & $70-80$ & Moderado \\
\hline $\begin{array}{c}>35 \% \\
2000-3000 \mathrm{~mL}\end{array}$ & $\begin{array}{c}\text { Letárgica } \\
\text { Inconciente }\end{array}$ & $\begin{array}{c}\text { Palidez, frialdad } \\
\text { más sudoración más } \\
\text { llenado capilar >3 } \\
\text { segundos }\end{array}$ & $>120$ & $<70$ & Severo \\
\hline
\end{tabular}

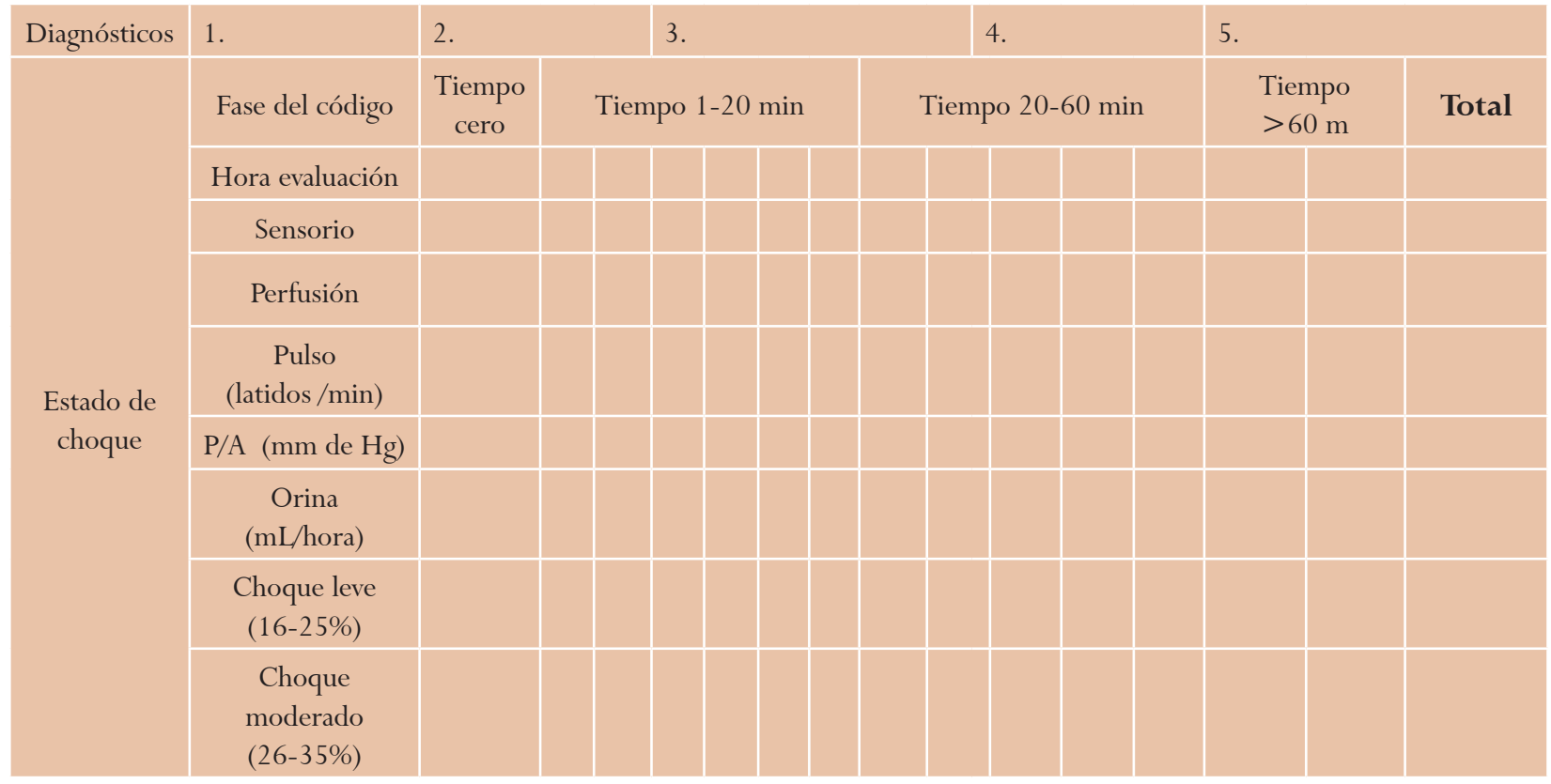




\begin{tabular}{|c|c|c|c|c|c|c|}
\hline Diagnósticos & 1. & 2. & 3. & 4. & 5. & \\
\hline \multirow{10}{*}{$\begin{array}{l}\text { Estado de } \\
\text { choque }\end{array}$} & Fase del código & Tiempo & Tiempo 1-20 min & Tiempo 20-60 min & $\begin{array}{l}\text { Tiempo } \\
>60 \mathrm{~m}\end{array}$ & \multirow[t]{2}{*}{ Total } \\
\hline & $\begin{array}{c}\text { Choque severo } \\
\qquad>35 \%)\end{array}$ & & & & & \\
\hline & $\begin{array}{c}\text { Solución salina } \\
0,9 \%\end{array}$ & & & & & \\
\hline & Hartmann & & & & & \\
\hline & $\begin{array}{c}\text { Glóbulos rojos } \\
\text { O (negativo) }\end{array}$ & & & & & \\
\hline & $\begin{array}{c}\text { Glóbulos rojos } \\
\text { O (positivo) }\end{array}$ & & & & & \\
\hline & Plaquetas & & & & & \\
\hline & Plasma & & & & & \\
\hline & Crioprecipitado & & & & & \\
\hline & Otros & & & & & \\
\hline \multirow{4}{*}{$\begin{array}{l}\text { Resultados } \\
\text { de la } \\
\text { coagulación }\end{array}$} & Plaquetas & & & & & \\
\hline & TP / TPT & & & & & \\
\hline & Fibrinógeno & & & & & \\
\hline & Dímero D & & & & & \\
\hline
\end{tabular}

Observaciones-eventos adversos: 